\title{
El entorno social de un escritor económico a comienzos del siglo XVII. El ejemplo de Alberto Struzzi ${ }^{1}$
}

\author{
Miguel Angel Echevarría Bacigalupe*
}

\section{The social environment of an economic writer at the beginning of the XVIIth Century. The Alberto Struzzi's exemple}

\begin{abstract}
RESUMEN The present paper analyzes the personality of Alberto Struzzi (1557-1638),

El presente trabajo analiza la figura de Alberto Struzzi (1557-1638), un hombre de negocios, escritor y diplomático italiano que residió en las principales ciudades de la Europa de su tiempo, como Bruselas, Praga o Madrid. Para ello distinguimos los círculos o esferas sociales que el autor frecuentó, en especial la familia, los negocios y las cortes principescas. Ello nos permitirá conocer mucho mejor las raíces de su pensamiento económico y social, así como el papel jugado por la baja y media nobleza en la primera Edad Moderna europea.

PALABRAS CLAVE: Alberto Struzzi, Bruselas, Praga, Madrid, mentalidad social, pensamiento económico.ABSTRACT

an Italian businessman, diplomat and economic writer who lived in the main European cities of the time like Brussels, Prague or Madrid. With this aim we have distinguished the circles (or social spheres) frequented by the author, especially his familial environment, his economic activities and the princely courts. It will allow to know better the roots of Struzzi's economic and social thought, as well as the role played by the small and middle nobility in the European Early Modern Times.

KEYWORDS:

Alberto Struzzi, Brussels, Prague, Madrid, social mentality, economic thought.

Recibido: 3-05-2010

Aceptado: 4-06-2010

* Universidad del País Vasco. Facultad de Ciencias Económicas y Empresariales. Depto. de Historia e Instituciones Económicas. Avda. Lehendakari Aguirre, 83 - 48006 Bilbao (Vizcaya) miguela.echeverria@ehv.es

1 El presente artículo constituye una versión resumida de la ponencia que presenté en el Curso de Verano de la UNED «El arte de la diplomacia en la Europa Moderna: un universo entre bambalinas» (julio de 2009). Agradezco a los dos evaluadores anónimos del texto sus juiciosos comentarios.
\end{abstract}




\section{INTRODUCCIÓN}

La forma de estudiar a un escritor económico y calibrar su importancia ha salido ganando en los últimos tiempos con una serie de métodos vinculados primordialmente al estudio de su entorno. Hay trabajos que nos presentan un amplio cuadro del modo de vida de dichos escritores: medio social, mayor o menor frecuencia, origen y distribución geográficos, media de obras que escribieron..., en fin, una visión coral sin duda interesante ${ }^{2}$, pero de escaso relieve por cuanto nos centramos aquí en una sola persona y su entorno inmediato. Mirado desde el plano individual, es indudable que la Sociología de Redes nos ha llevado a un campo de amplias posibilidades. Los sociólogos anglosajones han resaltado cuestiones como el impacto del entorno y el rol social que adquirimos en función de nuestros contactos, metodología que ha influido incluso en el terreno económico $^{3}$. Sin embargo, sabemos que la estructura social del Antiguo Régimen estaba fuertemente compartimentada, lo que facultaba o impedía determinados privilegios y actividades. Que esa coraza interestamental pudiera ser horadada no anula en modo alguno su existencia ni la obsesión de los coetáneos por mantenerla; sólo un uso muy intenso de la economía monetaria, junto con diversas crisis políticas y sociales de carácter antifeudal, abrieron el camino a una adecuación basada en la fortuna y no tanto en el origen. Por todo lo cual, el vínculo entre un escritor económico (concretamente, trataremos acerca del italiano Alberto Struzzi) y su contexto no puede simplificarse en un análisis de redes convencional, donde se privilegia la noción de vínculo sobre la de grupo y jerarquía, so pena de distorsionar la realidad sociológica de entonces.

¿Cómo proceder entonces? Dejémonos llevar por los coetáneos. El peso imponente de lo cosmológico (con sus dos grandes variantes, la astronomía y sobre todo la astrología) en su vida privada, llevaba al hombre moderno a concebir la realidad próxima como un universo de tamaño terrenal. Todo lo relativo al ser humano y a su entorno inmediato quedaba englobado bajo el concepto de microcosmia ${ }^{4}$; pues como dijera Baltasar Gracián, «es el hombre aquel célebre microcosmos" ${ }^{5}$. Lo mismo que en el Universo, las actividades y vivencias humanas se desarrollan dentro de círculos o esferas cuyas trayectorias o ámbitos, al engranarse, dan por resultado una armonía vital. Individuo, familia, grupo, clase, nación o cuerpo político, quedaban delimitados por espacios perfectos, como per-

2 Véase al respecto el trabajo modélico de THÉRÉ, C., «Le cadre d'existence des écrivains économiques», Cahiers Internationaux d'Histoire Economique et Sociale, 18 (1986), pp. 340-360, en especial pp. 341-345.

3 SEMITIEL GARCÍA, M., Social Capital, Networks and Economic Development, Chentelham, E. Elgar, 2006, p. 16. La influencia en la historiografía ha sido asimismo de algún relieve en los últimos años. Por poner dos casos, véanse las obras de EDELMAYER, F., Söldner und Pensionäre. Das Netzwerk PhiIlipps II. Im Heiligen Römischen Reich, Wien, Oldenbourg, 2002, y VVAA: Réseaux de correspondance à l'âge classique (XVIe-XVIIle siècles), Saint-Etienne, Université de Saint-Etienne, 2006.

4 Una guía para comprender el microcosmos humano lo hallamos en la obra de ROOB, A., El Museo Hermético. Alquimia \& Mística, Köln, Taschen, 2001, pp. 534-611.

5 GRACIÁN, B., El Discreto, Madrid, Eds. Atlas, 1969, p. 314. 
fectos eran los círculos 6 . Pues bien, analizaremos a Alberto Struzzi lo más cercanamente posible a su tiempo, es decir, en función de algunos grandes círculos concéntricos que acomodaron su vida, y dentro de los cuales resulta factible una vinculación social específica?

El primer círculo sería el familiar, en tanto que esposo, padre, hermano, etc., procurando la conservación y acrecentamiento del grupo. Lo cual engarza con el segundo círculo, el de cortesano, primero en Parma y luego en Bruselas, sin perder ojo a la Praga de Rodolfo II, para acabar su carrera en el Madrid de los Austrias. Más allá de este círculo se halla el de hombre de negocios, ya que cortesano y negociante son actividades indisolublemente unidas en él. Eso nos traslada armónicamente al cuarto círculo, el de pensador y humanista, "el círculo del alma" según lo denominara Gracián. No entraremos sin embargo allí; nos conformamos con efectuar un análisis de sus vinculaciones socioeconómicas y de las estrategias que implican.

Ese método se aplicará al estudio de las cartas que Struzzi recibió en el período 1599-1615. De toda esa correspondencia ${ }^{9}$ desecharemos la de tipo ideológico o político, por caer fuera de nuestro propósito en esta Ponencia. A cambio, se nos ofrece en aquellas páginas un retrato vívido del Flandes de los Archiduques, y parcialmente de las cortes imperial y española; además, como desgraciadamente los archivos italianos sufrieron muy serios daños durante la Segunda Guerra Mundial, los datos que contienen estas fuentes son de un alto valor histórico para completar el rompecabezas de las relaciones políticas de los Farnesio sin tener que conformarnos con el resumen que de los legajos italianos hiciera Ciriaco Pérez Bustamante $^{10}$.

Los hombres de la Edad Moderna ponían máxima atención al cultivo de sus relaciones epistolares. En este sentido, la historiografía ha resaltado con justeza el

${ }^{6}$ Resultaba ya clásico entonces comparar al monarca con el Sol, planeta que ilumina a los demás y que con el tiempo pasará a estar en el centro del cosmos político. Sobre este particular, véase el libro de MíNGUEZ, V., Los reyes solares. Iconografía astral de la Monarquía Hispánica, Castellón, Universitat Jaume I, 2001.

${ }^{7}$ Ello se refleja en el tratamiento que recibe de sus corresponsales al comienzo de las cartas, yendo de llustre Señor (distintivo de gentilhombre) a Muy llustre Señor, lo que denota haber alcanzado el grado de caballero. A tales denominaciones añade en su correspondencia italiana el calificativo de Osservandissimo; en las misivas españolas no falta tampoco el añadido «que Dios guarde». El status y el honor correspondiente debían quedar a salvo, incluyendo las cartas familiares, a las que se añade simplemente el grado parentesco (primo, tío, etc.)

${ }^{8}$ Para conocer el pensamiento struzziano, ECHEVARRIA BACIGALUPE, M. A., Alberto Struzzi, un precursor barroco del capitalismo liberal, Leuven, University Press, 1995.

${ }^{9}$ El grueso de la correspondencia, en Archives Générales du Royaume à Bruxelles (AGRB), Papiers Le Mesureur (PLM), leg. 9. Las misivas entre Ericio Puteano y Struzzi están editadas en ECHEVARRÍA BACIGALUPE, M. A., «Erycius Puteanus et Alberto Struzzi: histoire d'une amitié», Humanistica Lovaniensia, XLIX (2000), pp. 239-253, y en PUTEANUS, E., Erycii Puteani Epistolarum Promulsis, Lovaina, Ex Officina Flaviana, 1612, cartas XLVI y XLII, sin p.

10 PÉREZ BUSTAMANTE, C., La correspondencia diplomática entre los duques de Parma y sus agentes o embajadores en la corte de Madrid durante los siglos XVI, XVII y XVIII, Madrid, Tipografía de Archivos, 1934. 
papel vital de la correspondencia como medio de controlar el desarrollo de las campañas bélicas o atenuar la información imperfecta del mercado, cuando las comunicaciones eran difíciles y la expansión territorial convertía a la misiva en el único medio de asegurar el éxito de las estrategias o la rentabilidad inversora; míticos se hicieron algunos servicios de correos al servicio de los monarcas y negociantes más conspicuos del siglo ${ }^{11}$. Amsterdam, la gran plaza de negocios a comienzos del $\mathrm{XVII}$, basaba buena parte de su auge en el dominio de las comunicaciones epistolares con el exterior ${ }^{12}$, y lo mismo podríamos decir de otras ciudades clave como Sevilla, Lyon, Piacenza o Frankfurt.

Felizmente para el historiador, los comienzos del XVII conocieron un desarrollo espectacular de la comunicación epistolar, que en la Monarquía Hispánica se patentiza durante el reinado de Felipe $\mathrm{III}^{13}$.

\section{EL CÍRCULO FAMILIAR}

En este examen egocéntrico de la correspondencia de Struzzi, damos el primer lugar al círculo personal, compuesto por los lazos familiares. Alberto Cesare Strucciani (apellido cambiado por la familia en Struzzi) nació el 24 de abril de 1557 en San Bartolomeo della Ghiaia, cerca de Parma ${ }^{14}$. El padre pertenecía a la pequeña nobleza del ducado, emparentada como rama menor a los Palmia, sus parenti altolocati. Piero dedicó sus afanes más que nada a asuntos comerciales y bancarios $^{15}$, y si bien poseyó algunos pequeños lotes de tierra, la familia se perfila como netamente urbana. Alberto tuvo seis hermanos, de entre los que destaca Giovan$\mathrm{ni}$, el tercero por orden de edad. Este Giovanni fue notario, actividad en la que introdujo a su hermano menor, según parece como pasante ${ }^{16}$, aunque tales menesteres durarían poco en nuestro biografiado, ya que dejó su tierra natal para partir a los Países Bajos ${ }^{17}$. La documentación por mí manejada habla de Struzzi como colaborador asiduo del duque de Parma desde al menos 1578, año en que Alejandro Farnesio se hace con el cargo de Gobernador General de los Países Bajos ${ }^{18}$; sin

11 Es muy de destacar sin duda la familia Tassis, cuyo destino histórico en la Europa Moderna fue excepcional. Más adelante nos referiremos a ellos, porque la rama flamenca de la familia estaba entre las grandes amistades de Alberto Struzzi.

12 LESGER, C., The rise of the Amsterdam market and Information Exchange. Merchants, commercial expansion and change in the Spatial Economy of the Low Countries, 1550-1630, London, Ashgate, 2006, pp. 220-221.

13 MARTÍNEZ HERNÁNDEZ, S., «Significación y trascendencia del género epistolar en la política cortesana: la correspondencia inédita entre la infanta Isabel Clara Eugenia y el marqués de Velada», Hispania, LXIV, 217 (2004), p. 469.

${ }_{14}$ Los datos genealógicos de los Struzzi, en CADOPPI, A., «Notizie biografico-genealogiche su Alberto Struzzi (Parma 1557 - Madrid 1638)», Aurea Parma, LXXXVII, 1 (2003), pp. 123-140.

15 Ibid., p. 133. Piero falleció en 1573.

16 Ibid.

17 Giovanni murió en 1611, circunstancia a la que se alude en una carta de Domingo Vivanco a Struzzi, AGRB, PLM, leg. 9, fol.121, Madrid, 25 de septiembre de 1612.

18 ECHEVARRÍA BACIGALUPE, M. A., Alberto Struzzi..., p. 24. 
embargo, el escritor dejó la sede parmesana en marzo de $1581^{19}$. A partir de entonces participaría en los febriles aconteceres de la guerra al lado de su señor como guardarropa (lo que incluye la coordinación de su servicio de espionaje) ${ }^{20}$, habiendo logrado brillantes misiones que Alejandro elogia en documentos oficia$l \mathrm{es}^{21}$. Tras la muerte del duque de Parma, los años noventa parecen haber pasado en cierta penumbra para Struzzi. Llegado 1596, aparece en una Relación de la Casa del Archiduque Alberto de Austria, nuevo Gobernador General de Flandes, como "entretenido y criado de Su Alteza», con un sueldo de 25 florines entregado por el conde de Fuentes ${ }^{22}$. Es llamativo el comentario que se hace en dicha Relación: «Tiene muy ruin opinión con los naturales y con todo el exército»" ${ }^{23}$, lo que no menos sorpresivamente concuerda con el parecer que en 1603 se tiene del mismo archiduque Alberto, quien al decir de Cabrera de Córdoba, «está malquisto de los soldados y de la tierra", por lo que Felipe III pensaba enviarle a Borgoña con la infanta Isabel, tomando para sí la gobernación directa de Flandes ${ }^{24}$. Sea lo que fuere, a partir de entonces la relación de Struzzi con los archiduques Alberto e Isabel Clara Eugenia será muy estrecha. Posiblemente fuera una unión natural entre gentes más dotadas para la paz que para el fragor bélico.

Entre los otros parientes de la correspondencia, citaremos en primer lugar a Camillo Palmia, hijo de Giovanni Palmia (primo segundo de Alberto) y de Cecilia Cantelli, también originario de Parma ${ }^{25}$ y que se educa bajo los auspicios de Struzzi en la casa de Ericio Puteano, amigo íntimo del escritor. Las cartas de este humanista flamenco nos dan un cuidado retrato de la educación de Camillo al lado de sus hijos, aprendiendo latín y lengua flamenca con aplicación ${ }^{26}$. Struzzi y Puteano se habían encargado de la educación de jóvenes de familias nobles y de la alta burguesía, tanto más cuanto que en este caso se trata de un miembro del tronco familiar de los Palmia. La lista de parientes de sangre se cierra con Isabella Struzzi, sobrina de Alberto como hija de Giovanni; tras su entrada en el convento de San Alessandro de Parma tomará el nombre de Brígida, con el que aparece en las cartas $^{27}$.

Alberto Struzzi estuvo casado dos veces. La primera, con Luisa Haller von HaIlerstein, lo que le pone en contacto con los poderosos Haller von Hallerstein, fa-

19 CADOPPI, A., op. cit., p. 135. Struzzi habría hecho previamente testamento.

20 AGRB, Papiers d'Etat et de l'Audience (PEA), leg. 33/4, sin fol. Lista de personas de la casa del duque de Parma, Bruselas, 24 de enero de 1586.

21 ECHEVARRÍA BACIGALUPE, M. A., Alberto Struzzi..., p. 24.

22 Archivo General de Simancas (AGS), Secretaría de Estado (Estado), leg. 612, sin fol.

23 Ibid.

24 CABRERA DE CÓRDOBA, L., Relaciones de las cosas sucedidas en la corte de España desde 1599 hasta 1614 (R. García Cárcel, Ed.), Salamanca, Junta de Castilla y León, 1997, p. 174.

25 Nació el 12 de diciembre de 1605; CADOPPI, A., op. cit., p. 130.

26 ECHEVARRÍA BACIGALUPE, M. A., Erycius Puteanus..., pp. 251-252.

27 En una carta de agosto de 1613, Brígida manifiesta su pésame por la muerte de Dorotea Romeu, segunda esposa de Struzzi, AGRB, PLM, leg. 9, fol. 166. En 1614 envía dos cartas a Alberto pidiéndole dinero, AGRB, PLM, leg. 9, fols. 175-177, 28 de enero y 8 de abril. Su tono no puede ser más obsequioso, declarándose siempre «nipote e serva affettissima», aunque más que suplicar, exige. 
milia alemana establecida en Amberes pero con raíces en Nuremberg (ciudad imperial de mayoría protestante donde los Haller llegaron a ser alcaldes vitalicios), y cuya fama y fortuna en la Europa del XVI se basaron en la alianza con los Fugger para consentir préstamos a la Casa de Austria ${ }^{28}$. Las misivas apenas registran su paso fugaz, siempre en la parte final del besamanos, y sólo entre 1603 y 1608; después desaparece cualquier alusión a su persona ${ }^{29}$. Sabemos que Struzzi se hallaba en Nuremberg en 1601 y 1602; para mediados de 1603 sus corresponsales le escriben a Bruselas ${ }^{30}$, lo que explica la orden de vender en Parma el resto de sus bienes, y la obtención en 1604 de una carta de burguesía para residir en la capital de los Países Bajos meridionales ${ }^{31}$, donde su esposa poseía dos casas. Eso pondrá asimismo inicio a su actividad como representante de Ranuccio I ante los Archiduques, e indirectamente en la corte de Madrid.

Son pues años de intensa dedicación profesional en Nuremberg que le llevan incluso hasta Praga ${ }^{32}$, pues él también es otro proveedor de capitales al Imperio. Paralelamente, hasta el final de sus días peleará por cobrar las importantes sumas que el rey de España adeuda a la familia Haller, de las que recibirá ciertas cantidades, aunque con la parsimonia que la Real Hacienda utiliza para sus acreedores $^{33}$.

Habiendo enviudado, la descendencia que no hallara con su primer matrimonio $^{34}$ Struzzi espera conseguirla en unas segundas nupcias con Dorotea Romeu, una joven valenciana dama de honor de la archiduquesa Isabel Clara Eugenia ${ }^{35}$. La boda tendrá lugar en junio de 1612, habiendo cumplido ya Struzzi los 55 años. Esto nos habla otra vez de una estrategia para reforzar los vínculos con la corte archiducal, alejándose de un Ranuccio Farnesio cuya estrella declinaba a ojos vista en Madrid y Bruselas.

El matrimonio con Dorotea es de los asuntos que más testimonios han dejado en la correspondencia que manejamos, quizá porque paralelamente a su boda vino el cargo de caballerizo del Archiduque. El nacimiento de un hijo (Eugenio Jusephe)

28 KELLENBENZ, H., Los Fugger en España y Portugal hasta 1560, Salamanca, Junta de Castilla y León, 2000, pp. 566 y 603; EHRENBERG, R., Le siècle des Fugger, Paris, SEVPEN, 1955, pp. 118-119; ECHEVARRÍA BACIGALUPE, M. A., Alberto Struzzi..., pp. 21-22.

29 La primera referencia a Luisa Haller la hallamos en una carta de Guillén de San Clemente fechada en Praga el 4 de mayo de 1602, AGRB, PLM, leg. 9, fol. 264; la última es la de fray Juan Neyen, escrita el 20 de agosto de 1608, ibid., fol. 63.

30 «He savido de su buena llegada a esse lugar y de Madama con salud, de que les doy la norabuena». Guillén de San Clemente a Struzzi, AGRB, PLM, leg. 9, fol. 266, Praga, 21 de junio de 1603.

31 ECHEVARRÍA BACIGALUPE, M. A., Alberto Struzzi..., p. 16.

32 La estancia de Struzzi en la corte checa, aludida en carta de Guillén de San Clemente, ARGB, PLM, leg. 9, fol. 294, Praga, 26 de febrero de 1605.

${ }_{33}$ ECHEVARRÍA BACIGALUPE, M. A., Alberto Struzzi..., p. 34.

${ }_{34}$ Aunque sí riqueza material, como las dos casas de Bruselas, bienes inmuebles en Nuremberg, objetos de valor y otros, por un montante de 66.000 florines; ibid.

${ }^{35} \mathrm{El}$ apellido Romeu (Romero) es de origen aragonés. Extendido por Cataluña y el reino de Valencia, se distinguió durante la Reconquista. GONZÁLEZ-DORIA, F., Diccionario heráldico y nobiliario de los Reinos de España, Madrid, Trigo Eds., 2000, p. 725. 
tuvo lugar a mediados de 1613 , de lo que dan fiel testimonio las cartas ${ }^{36}$. Pero Dorotea no sobrevivió al parto. De ese luctuoso acontecimiento nos enteramos por otra serie de misivas, nueva ocasión para mostrar el sólido entramado de los vínculos sociales de Struzzi ${ }^{37}$.

La correspondencia sobre Dorotea Romeu encierra, más allá de felicitaciones y pésames, algunos datos sobre coyuntura económicosocial dignos de tenerse en cuenta. Me refiero especialmente a la carta de Domingo Trobado (tío de Dorotea) enviada a Alberto Struzzi el 8 de marzo de 1613, y que en pocas frases lapidarias refleja el hondo malestar reinante, sobre todo en la región valenciana. Primeramente, retrata la coyuntura general con un lamento: "Cualquier cossa que se mueva es de grande trabaxo para Espanna según está desnerviada de gente y de dinero», porque el país «no ha luzido después de la muerte de Filipo Segundo»38. El Prudente comienza a ser añorado no mucho después de su fallecimiento por unas gentes que en vida de aquél se quejaban de su gobierno en términos tan alarmantes como reflejan por ejemplo las actas de las Cortes de Castilla ${ }^{39}$. Pero la coyuntura desfavorable suele hacer buenos a los que la preceden, y toda una serie de biografías sobre Felipe II aparecidas desde comienzos del XVII, retratan al monarca como un espejo en que debían mirarse los contemporáneos.

Domingo Trobado menta en su carta los tristes destinos de un Reino de Valencia otrora pujante y hoy presa de corsarios berberiscos ayudados por holandeses, que no cejan en sus correrías por la costa ${ }^{40}$. Recela incluso una guerra civil a causa de las fuertes imposiciones fiscales ${ }^{41}$. En definitiva, tanto ha cambiado Valencia, que quienes se hayan ausentado de ella por algún tiempo, no podrán ya reconocerla. La investigación histórica nos ha descrito con detalle los trastornos que supuso la expulsión del grupo morisco y la repoblación hecha por los cristianos, que se tradujo en caída de productividad y por ello de rentas, con miseria, hambre y delincuencia generalizadas. Aunque dicho panorama no puede achacarse exclusivamente a la salida de los exiliados ${ }^{42}$, la extensión del bandolerismo y la multiplicación de toda clase de tropelías harán de la vida cotidiana una dura su-

${ }^{36}$ AGRB, PLM, leg. 9, con cartas del conde de Elberg (fol. 110), de Francesco Pegoloti (fol. 113), Brígida Struzzi (fol. 115), fray Iñigo de Brizuela (fol. 136), conde de Añover (fol. 143) y de Catalina, hermana de Dorotea (fol. 161).

${ }^{37}$ Los mismos le escriben poco después con las condolencias de rigor; ibid., fols. 43, 144, 147, 155 , 160, y 166.

38 Ibid., fol. 132.

39 Los compromisos bélicos en el exterior sirvieron frecuentemente de polémica en las sesiones de Cortes, ECHEVARRÍA BACIGALUPE, M. A., «Las últimas Cortes del reinado de Felipe II (1592-1598)», Estudios de Deusto, XXXI, 2 (1983), pp. 337-340.

40 AGRB, PLM, leg. 9, fol. 132. El Consejo de Estado retrata fielmente en 1611 la relación entre moriscos y rebeldes holandeses para atacar las costas españolas, a pesar de la tregua firmada en 1609; AGS, Estado, 634, sin fol., Madrid, 31 de julio de 1611.

41 AGRB, PLM, leg.9, fol. 132: «Temo alguna disensión civil entre ciudadanos respective de cada ciudad por gabelas y pechos excesivos que cada día se ponen».

42 ARDIT, M., «Una reflexión sobre la expulsión de los moriscos valencianos y la repoblación», Revista de Historia Moderna, 27 (2009), p. 306. 
pervivencia, coadyuvando a mantener el estancamiento ${ }^{43}$. El malestar, acumulado durante décadas, acabará estallando en la Segunda Germanía (1693) ${ }^{44}$.

\section{EL CÍRCULO DE LA CORTE}

La corte, por muchos comparada a un pequeño universo en sí misma, toca tangencialmente el microcosmos vital de Alberto Struzzi. Con origen en Parma, su experiencia cortesana pasará por las principales ciudades europeas de entonces. Conocemos los inicios a las órdenes de Alejandro Farnesio, y cómo pasa luego al servicio de Alberto de Austria, decidiendo quedarse definitivamente en Flandes. En el período 1603-1614, Struzzi simultanea sus actividades de hombre de negocios y de corte en los Países Bajos con la representación de los intereses de Ranuccio I Farnesio, duque de Parma (1569-1622), sustituyendo a Cosimo Masi ${ }^{45}$. Ranuccio pretendió mucho más de lo que su poder territorial y político permitía. Fue hecho caballero de la orden del Toisón de Oro en 1601 de manos de su tío Felipe III46, $^{46}$ junto con una pensión de quince mil escudos anuales ${ }^{47}$. Asimismo hizo de padrino en el bautismo de la infanta Ana Mauricia ${ }^{48}$. Sus miras iban hacia el virreinato de Portugal, que sin embargo no obtuvo. Quiso después acceder al puesto de Gobernador General de Flandes en sustitución de los Archiduques, también sin éxito ${ }^{49}$. Pero los grandes sinsabores le llegaron de Italia: el conde de Fuentes, gobernador de Milán, le tomó el marquesado de Novara, siguiendo de 1600 a 1604 varias cuestiones fronterizas entre Parma y el ducado milanés ${ }^{50}$; en 1616, Ranuccio aún solicitaba a Madrid se le retornase Novara ${ }^{51}$. En cuanto al gobierno interior del ducado, usó con frecuencia de métodos expeditivos, llegando a ajusticiar en 1612 a los principales representantes de la vieja nobleza, poniendo punto final a la lucha de largos años entre los advenedizos Farnesio y familias de más antiguo blasón ${ }^{52}$. Podemos incluir a nuestro escritor entre los «homines novi» de Parma sa-

43 FELIPO, A., El centralismo de nuevo cuño y la política de Olivares en el País Valenciano. Fiscalidad, control político y hacienda municipal (1621-1634), Valencia, Ayuntamiento, 1988, pp. 133 y 135.

44 Sobre la decadencia valenciana, CASEY, J., El Reino de Valencia en el siglo XVII, Madrid, Siglo XXI, 1983, caps. 3 a 6 . A tener en cuenta igualmente la importante aportación historiográfica de GARCÍA MARTÍNEZ, S., Valencia y la Casa de Austria, Valencia, Anubar, 1977, y Bandolers, corsaris y moriscos, Valencia, Eliseu Climent, 1980.

45 ECHEVARRÍA BACIGALUPE, M. A., Alberto Struzzi..., p. 26.

46 CEBALLOS-ESCALERA Y GILA, A., La Insigne Orden del Toisón de Oro, Madrid, Palafox \& Pezuela, 2000, p. 310.

47 LHERMITE, J., El Pasatiempos, Madrid, Eds. Doce Calles, 2005, p. 592.

48 Para la descripción del bautismo, ibid., pp. 582-586.

49 PÉREZ BUSTAMANTE, C., op. cit., p. 38.

50 Ibid., p. 26. El marquesado era dominio de los Farnesio junto con los ducados de Parma y Plasencia, los ducados de Castro y Ronciglione, y algunos feudos en el reino de Nápoles.

51 PEREZ BUSTAMAMTE, C., op. cit., p. 40.

52 ROMANI, M. A., «Finanza pubblica e finanze del Principe nella formazione dello Stato Farnesiano di Parma e Piacenza (1545-1612)», en Estado y Fiscalidad en el Antiguo Régimen, (C.M. Cremades Griñán, Ed.), Murcia, Universidad de Murcia, 1989, p. 191. 
lidos de la nobleza pequeña y media, opuestos al poder de los grandes tradicionales ${ }^{53}$.

Pero la Parma de Ranuccio Farnesio fue perdiendo peso entre los intereses de Struzzi. Desde 1601 intensifica su relación con los archiduques Alberto e Isabel. Como asentista y miembro de la familia Haller, deseaba cobrarse los empréstitos consentidos a la monarquía hispana, así que los Archiduques, por sus buenos contactos con el duque de Lerma ${ }^{54}$, suponían una vía primordial de acercamiento al entorno de Felipe III. Otro personaje cercano a la estela de Lerma era el conde de Fuentes, gobernador del Milanesado; a pesar de sus diferencias con Ranuccio, Fuentes era un objetivo político a conseguir. La relación con Praga viene tanto de considerandos políticos (estar cerca del Emperador) como económicos (finanzas). Con todo ello, Struzzi cerraba un triángulo estratégico cuyo circuncentro será Bruselas y los vértices Madrid, Milán y Praga, con prolongaciones hacia Italia (Parma, Roma) y Alemania (Nuremberg); dicho triángulo, que corresponde a las áreas de poder Habsburgo, se inscribe perfectamente en su círculo cortesano, dándole sentido.

Pasemos a dar pruebas de esos contactos por las cuatro capitales. Bruselas y Mariemont (residencia favorita de Isabel Clara Eugenia para el buen tiempo) centran la vida cortesana flamenca; el balneario de Spa (Lieja), se convierte paralelamente en un punto de encuentro internacional muy frecuentado. La correspondencia a Struzzi que manejamos nos indica frecuentemente la llegada de las cartas desde esos lugares. Entre los amigos con los que cuenta el de Parma sobresale el sumiller de corps don Rodrigo Niño y Lasso, conde de Añover, encargado por Felipe III de asesorar al archiduque Alberto en el gobierno de Bruselas y procurar el enderezamiento de las cosas militares, en las que aquél no destacaba ${ }^{55}$. Para 1612, Añover llegará a ser simultáneamente Mayordomo Mayor, lo que equivale a decir confidente del Archiduque. Ello implica, entre otras cosas, supervisar las actividades secretas, de ahí su control de espías como Manuel López y Emmanuel Sueyro. Alberto Struzzi, agente de inteligencia nunca retirado, tomará la defensa de los Sueyro años después contra los ataques del cardenal de La Cueva ${ }^{56}$. Añover murió en 1620, cuando nuestro escritor ya estaba en España ${ }^{57}$.

53 Ibid.

54 Buen entendimiento que se evidencia en las misivas, especialmente por parte de la infanta, RODRíGUEZ VILLA, A., Correspondencia de la Archiduquesa doña Isabel Clara Eugenia con el duque de Lerma y otros personajes, Madrid, 1906. No así de buenas serían inicialmente las relaciones de Isabel Clara con Olivares, FERNÁNDEZ LARRAIN, S., La rendición de Breda, Madrid, Editora Nacional 1968, pp. 44-45.

55 ECHEVARRÍA BACIGALUPE, M. A., Flandes y la Monarquía Hispánica, 1500-1713, Madrid, Sílex, 1998 , p. 179.

56 Biblioteca Nacional de Madrid (BNM), ms. 10.441, fol. 268 v. Los problemas de los Sueyro con La Cueva, en ECHEVARRÍA BACIGALUPE, M. A., La diplomacia secreta en Flandes, 1598-1643, Leioa, UPV, 1984, pp. 180-182.

57 MARTÍNEZ HERNÁNDEZ, S., op. cit., p. 499. 
Ambrosio Spínola, estrella rutilante en la corte archiducal, no podía quedar ajeno a la red de alianzas struzziana. Por alusiones de fray Juan Neyen, sabemos del buen entendimiento entre ambos ${ }^{58}$, lo que se confirma en la dedicatoria de un libro de Struzzi ${ }^{59}$. Normal que fuera así en dos personas unidas por el origen geográfico, el status nobiliario, la dedicación a los negocios y, a pesar del generalato, una firme vocación por la paz, fomentadora de negocios. Luego conocemos la amistad del escritor, por vía de Dorotea Romeu, con varios miembros influyentes de la corte archiducal $^{60}$ que le abrieron el camino hacia los cargos palaciegos.

Las relaciones de Struzzi con el alto clero fueron asimismo frecuentes. A destacar fray Iñigo de Brizuela, confesor de Alberto y por ende gran personaje de la corte. Con las recomendaciones del Archiduque, se envió al religioso en 1607 para tratar de una posible tregua con los rebeldes, repitiendo viaje al año siguiente ${ }^{61}$ y poniendo en evidencia la absoluta necesidad de una suspensión de hostilidades, siquiera por motivos presupuestarios ${ }^{62}$. También fue delegado en 1607 y 1608 fray Juan Neyen, natural de Amberes y Comisario General de los frailes franciscanos observantes en los Países Bajos. Aunque la acogida en Madrid fue inicialmente bastante fría, si atendemos a la correspondencia que Neyen envía a Struzzi, el religioso logró audiencia de Lerma, de Felipe III y del Consejo de Estado ${ }^{63}$. Parece que sus conversaciones surtieron efecto, pues se envió como embajador en Bruselas al marqués de Guadalest en sustitución de don Diego de Ibarra, para coordinar con Ambrosio Spínola la estrategia de cara a una tregua con los rebeldes ${ }^{64}$.

Estas relaciones con el clero se extienden a la nunciatura en Bruselas. El primer nuncio, Ottavio Mirto Frangipani, sólo dejó deudas impagadas tras su marcha a Tarento como arzobispo en 1608. Los esfuerzos de Struzzi por recuperar lo prestado han dejado huella en los archivos ${ }^{65}$, pero la muerte del nuncio-arzobispo en 1612 frustró la posibilidad de un arreglo.

La cohesión lograda en los Países Bajos quiere Struzzi extenderla a otras áreas. De ahí que la correspondencia salte hacia Milán, donde desde 1600 go-

58 Struzzi y su mujer se hallaban enfermos en 1608, y Neyen espera que Spinola les haga una visita para consolarles en la tribulación, AGRB, PLM, leg. 9, fol. 63, Valladolid, 20 de agosto de 1608.

59 Se trata de la obra Imagen de una milicia y de un ejército firme a favor del marqués Ambrosio Spinola, Bruselas 1614.

60 Aparecen nombres relevantes (además del ya mencionado Añover) como Juana de Jasincourt, dama de Isabel Clara, el marqués de Billars, el de Guadalest (diplomático español), Ottavio Visconti (caballerizo mayor), Philippe de Croy, conde de Solre, gentilhombre de cámara de Alberto y como Struzzi escritor económico, o el conde de Bucquoy, también gentilhombre de cámara del Archiduque.

61 AUSTRIA, A. DE, “Cartas al duque de Lerma», CODOIN, tomo XLIII, Madrid 1863, pp. 22, 144, y 146-148. Sobre el papel de Brizuela, RODRÍGUEZ VILLA, A., op. cit., cap. XVI.

62 AGS, Estado, leg. 626, sin fol. Informe de fray Iñigo de Brizuela a Felipe III, año 1608.

63 AGRB, PLM, leg. 9, fol. 45, Madrid, 10 de agosto de 1607. Respecto a Lerma, afirma Neyen: «Puedo asegurar a Vuestra Señoría que tengo un padre en él», ibid. Dice asimismo que habló a solas con el rey, y que luego tuvo reunión con el Consejo de Estado, presidido por Lerma.

64 Acerca del papel de Neyen, RODRÍGUEZ VILLA, A., Ambrosio Spínola, primer marqués de Los Balbases. Ensayo biográfico, Madrid 1904, cap. XIV; RUBIO, J. M., Los ideales hispanos en la tregua de 1609 y en el momento actual, Valladolid, 1937, pp. 53 y 66-70.

65 AGRB, PEA, leg. 247, fols. 173, 231 y 240. 
bierna en nombre de Felipe III don Pedro Enríquez de Guzmán, conde de Fuentes. Aunque gran enemigo de los Farnesio, como ya se ha visto, no obstará para que Struzzi labre unas excelentes relaciones con Fuentes para asegurarse la protección española en Italia e indirectamente el apoyo de Madrid ${ }^{66}$; además era el intermediario ideal para defender los intereses de Ranuccio I sin enemistarse con ninguno de ellos.

Punto clave en la Europa de entonces lo constituía la corte imperial, sita en Praga por voluntad de Rodolfo II, lo que le alejaba de los turcos y de la nobleza austríaca, no menos enemiga. Allí, y salvo algunos intervalos, don Guillén de San Clemente venía representando desde 1581 al rey de España ${ }^{67}$, habiendo cumplido importantes cometidos ${ }^{68}$. A Struzzi lo conocía por lo menos desde 1588 en calidad de proveedor habitual de cuadros $^{69}$. San Clemente fue ayudado en sus cometidos por Arnaldo van der Boye en calidad de secretario de lenguas y negocios, un puesto que ya ocupara desde 1580 al servicio del anterior embajador, don Juan de Borja ${ }^{70}$.

Praga vivió en torno al 1600 un ambiente febril, donde legados de todo el orbe cristiano y aún de los infieles se codeaban con miembros de palacio, alquimistas, filósofos, literatos, artistas y científicos, pero también con charlatanes, embaucadores, magos o espías. El palacio imperial y la ciudad de Praga fascinan aún hoy por la brillantez de sus logros intelectuales y artísticos; justo es decir que el diplomático español también se hizo notar por su pequeña corte paralela ${ }^{71}$. Tras su muerte en 1608, San Clemente será sustituido por don Baltasar de Zúñiga, otro experto en temas flamencos ${ }^{72}$.

66 Se decía en los medios cortesanos que a Lerma y Fuentes les vinculaba sólida alianza, GÓMEZ RIVERO, R., op. cit., pp. 200-201. Sin embargo, lo desmiente CONTARINI, S., Estado de la Monarquía Española a principios del siglo XVII (J. Gil Sanjuán, Ed.), Málaga, Ed. Algazara, 2001, p. 56.

67 Con la excepción más importante entre 1595 y 1599, según AYERBE, M. DE, «Introducción», en Correspondencia inédita de Guillén de San Clemente, embajador en Alemania de los reyes don Felipe II y III sobre la intervención de España en los sucesos de Polonia y Hungría, 1581-1608, (M. de Eyerbe, Ed.), Zaragoza, 1892, pp. IX-X. El embajador escribe a Struzzi desde la capital imperial en términos que denotan estar residiendo allí desde tiempo atrás; AGRB, PLM, leg. 9, fol. 251, Praga, 17 de marzo de 1601.

68 Para la trayectoria diplomática de San Clemente, OCHOA BRUN, M. A., Historia de la diplomacia española. La diplomacia de Felipe II, Madrid, Ministerio de Asuntos Exteriores, 2000, pp. 142-144, y CHUDOBA, B., España y el Imperio (1519-1643), Madrid, Sarpe, 1986, pp. 153-154.

69 GORIS, J.A., Etude sur les colonies marchandes méridionales (Portugais, Espagnols, Italiens) à Anvers de 1488 à 1567, Louvain, 1925, pp. 142-143.

70 AGS, Estado, leg. 2494, sin fol. Relación del año 1608.

71 ECHEVARRÍA BACIGALUPE, M. A., «Vida y muerte de las bibliotecas en los Países Bajos españoles (siglos XVI-XVII)», Opera Romanica, 9 (2006), p. 329; idem, «Economía y Revolución Científica en Europa, 1580-1610», Torre de los Lujanes, 65 (2009), pp. 164-165; MOLAS I RIBALTA, P., Cataluynya i la Casa d'Austria, Barcelona, Curial, 1966, p. 34; JIMENEZ DÍAZ, P., El coleccionismo manierista de los Austrias entre Felipe II y Rodolfo II, Madrid, Sociedad Estatal para la Conmemoración de los Centenarios de Felipe II y Carlos V, 2001, p. 126.

72 Zúñiga ofrece una descripción de los últimos días de San Clemente en AGS, Estado, leg. 2492, sin fol., Relación a Felipe III, 5 de septiembre de 1608. Allí comenta que su predecesor, antes de morir, «tenía hecho su testamento y ordenadas sus cosas, y ajustadas sus quentas», ibid. 
La correspondencia con Praga forma una carpeta aparte en el legajo ${ }^{73}$, y se divide en dos clases: las cartas enviadas por Van der Boye (1603-04), y las del propio embajador (1601-05). Geográficamente, unas se enviaron a Nuremberg (años 1601-02) y otras a Bruselas (1603-05), en función obviamente de la residencia habitual de Struzzi. Las de Van der Boye tienen relación con los intentos del italiano para ganarse el favor de Rodolfo II y gestionar los préstamos a cortesanos en apuros. En cuanto a San Clemente, sus cartas tienen siempre un tono de respetuosa cercanía, y se refieren tanto a demandas de productos, como al apoyo a las aspiraciones de Struzzi en la corte imperial, o a lograr componendas en el interior de la familia Tassis, correos mayores en Amberes $^{74}$.

Con Madrid, los vínculos eran aún relativamente lejanos. El abogado Francisco Obispo (o Francesco Lo Obispo) miraba los intereses de Struzzi en la capital hispana ${ }^{75}$, a lo que se acompañaban instancias ocasionales como la del padre Neyen, que se entrevistó con el secretario Andrés de Prada para tratar de obtener el pago de los asientos concedidos por Struzzi y los suyos tras la suspensión de pagos de $1607^{76}$. Luego están las gestiones del conde de Añover en su favor. Pero en conjunto, Madrid sólo aparece ocasionalmente en la correspondencia. Hay que esperar a 1614 para que la dinámica cambie, debido al envío de un regalo de los Archiduques al infante Felipe, consistente en un enano y un ejército de juguete, de cuyo transporte se encargó el mismo Struzzi el 11 de noviembre de aquel año ${ }^{77}$. Tal circunstancia le permitió tomar contacto directo con la corte española, en la que residiría de una manera mucho más estable hasta su fallecimiento ${ }^{78}$. ¿Por qué se quedó en España? Primero, por sus intereses personales (deudas de la Real Hacienda); luego, por representación de los Archiduques; después por la delicadísima situación en Italia a causa del conflicto contra Saboya ${ }^{79}$. Yo no desesti-

73 AGRB, PLM, leg. 9, fols. 250-304.

74 Para estos temas, ibid., fols. 293-295 (26 de febrero de 1605), fols. 296-298 (26 de marzo de 1605), fols. 299-301 (2 de abril de 1605), y fols. 302-304 (23 de abril de 1605).

75 Las relaciones Struzzi-Obispo, en AGRB, PLM, leg. 11, sin fol.

76 La referida carta de Neyen, en AGRB, PLM, leg. 9, fols. 44-46, Madrid, 10 de agosto de 1607. El rey ha decidido por el momento no pagar deudas «que se han hecho setenta y tantos años ha», y abona sólo las más recientes. En cuanto a Andrés de Prada, era experto en temas de Flandes desde tiempos de don Juan de Austria; CONTARINI, S., op. cit., p. 59; DEL RÍO, M. A., La crónica sobre don Juan de Austria y la guerra en los Países Bajos (1576-1578) (M. A. Echevarría Bacigalupe, Ed.), Wien, Oldenbourg, 2003, pp. 268-269.

77 Miguel Soplillo llegó para suceder al fallecido Bonami. Acerca del viaje, el enano y el ejército de figuras, ECHEVARRÍA BACIGALUPE, M. A., Alberto Struzzi..., pp. 17-19. Aquí añadiremos como novedad la insistencia del padre de Soplillo en pasar a vivir con su hijo a España (AGRB, PLM, leg. 9, fol. 179, Añover a Struzzi, 31 de agosto de 1614). También son destacables los problemas para publicar el ya citado opúsculo sobre el juguete (ECHEVARRÍA BACIGALUPE, M. A., Erycius Puteanus..., p. 253), como asimismo los obstáculos de Struzzi para componer en Madrid «la machina del exercito», AGRB, PLM, leg. 9, f. 195, Struzzi a G. Tibanti, 10 de enero de 1615.

78 Struzzi rompió en 1614 con Ranuccio, y ese mismo año vendió pertenencias suyas antes de pasar a Madrid, ciudad que apenas abandonaría ya. AGRB, PLM, leg. 12, sin fol., y AGS, Contadurías Generales (CCGG), leg. 200-1, sin fol.

79 BOMBÍN PÉREZ, A., La cuestión de Monferrato (1613-1618), Vitoria, Colegio Universitario de Alava, 1975, p. 241. 
maría tampoco la confirmación en 1613 de que Alberto e Isabel ya no podrían tener descendencia, y asimismo los esfuerzos para propiciar el mantenimiento de la tregua cuando ésta llegase su término.

Alberto Struzzi es indudablemente un entendido en bellas artes y música, condición sine qua non para ser buen cortesano, tal y como indicara Castiglione en su célebre obra ${ }^{80}$. En cuanto a la música, no dejó de proveer a la corte bruselense de cantantes e instrumentos músicales ${ }^{81}$. Con cierta frecuencia recibe encargos de adquirir cuadros, y como era de esperar, Struzzi poseyó en casa una variada colección de pinturas ${ }^{82}$. Sólo que él no piensa generalmente en el honor sino en el provecho, porque a diferencia de la nobleza de alcurnia, vende sus servicios como el negociante que es.

El diplomático y cortesano es el círculo de más relieve en la vida del autor parmesano junto al de las transacciones económicas, que pasamos a relatar ahora.

\section{EL CÍRCULO DE LOS NEGOCIOS}

En sus actividades profesionales como en sus informes, menudean las alusiones a los mecanismos feriales. Tuvo mucha fortuna en ello, porque las ferias de Piacenza eran las más importantes de la Europa de entonces y pertenecían jurisdiccionalmente al duque de Parma. Se les llamaba «ferias de Besançon» (de Bisenzone, en italiano), siendo creadas por los financieros genoveses en 1536, aunque su importancia sólo crecería desde 1579, al compás de las necesidades de la Real Hacienda española. Allí se canalizaban las expediciones de metal precioso hacia los Países Bajos, y cuando era posible, se cambiaba la plata por oro. El mecanismo permitió fijar para los efectos unos plazos de vencimiento muy estables, de unos dos meses ${ }^{83}$. Las ferias, corazón económico de todo Occidente ${ }^{84}$, permanecerán en el sitio hasta 1621. La moneda de aquella feria -el scudo di marchemantuvo una equivalencia estable con las monedas reales, por lo que se hizo referencia común a muchos europeos, como el mismo Struzzi señala ${ }^{85}$. Ello permitió

80 CASTIGLIONE, B., El Cortesano, 3aㅡ ed., Madrid, Espasa-Calpe, 1967, pp. 69 y 71.

81 Presenta en la corte a una muchacha con dotes para el canto, y manda fabricar un clavicordio por encargo del archiduque Alberto, AGRB, PLM, leg. 9, f. 206, 210 y 216, C. Licia a Struzzi, Mariemont, año 1612.

82 ECHEVARRÍA BACIGALUPE, M. A., Alberto Struzzi..., p. 37. En la correspondencia que manejamos suele alabarse el buen gusto artístico del escritor.

83 VÁZQUEZ DE PRADA, V., Lettres marchandes d'Anvers, Paris, SEVPEN, 1961, tomo I, p. 118.

${ }^{84}$ BRAUDEL, F., La Mediterranée et le monde mediterranéen à l'époque de Philippe II, Paris, Armand Colin, 1979, tomo I, pp. 348 y 458-460. Las ferias eran cuatro al año: la Aparición (1 de febrero), Pascua (2 de mayo), 1 de agosto, y Todos los Santos (2 de noviembre). Una visión actualizada sobre el mecanismo de estos centros, en PEZZOLO, L. y TATTARA, G., «Una fiera senza luogo: Was Bisenzone an International Capital Market in the Sixteeenth Century?», The Journal of Economic History, 68, 4 (2008), pp. 1098-1122.

85 El escritor resalta el valor referencial de las monedas usadas «en las ferias como en la de Plasencia, ciudad del duque de Parma», BNM, ms. 10.441, fol. 222 v, informe de Struzzi, circa 1623-25. 
ciertamente efectuar importantes negocios vinculados a la especulación entre la largueza y estrecheza de escudos di marche en Piacenza y cada una de las otras plazas ${ }^{86}$. Es la vía que utiliza nuestro autor, sea por motivo de negocios o para percibir los envíos regulares que hacen las familias de protegidos suyos en los Países Bajos, tales como los Torello y los Pallavicino ${ }^{87}$. A veces incluso el mismo Struzzi adelanta el dinero para cobrarse después en las ferias, según los tipos de cambio de Amberes-Piacenza y viceversa ${ }^{88}$. Caso de ignorar los tipos cambiarios al uso, consulta a amigos o proveedores, destacando Francesco Serra, conocido banquero de Amberes ${ }^{89}$. No menos conocedor fue del mundo de los negocios en Alemania, especialmente desde que emparentó con los Haller. Pero cobrar los empréstitos será un largo e infructuoso camino lleno de circunstancias desagradables. Por eso, suspensiones de pagos como las de 1596, 1607 y 1617, fueron duros golpes a la esperanza de reembolso, y aún se repetirán más tarde ${ }^{90}$. Familias conocidas como los Fugger ${ }^{91}$ o los Vergano ${ }^{92}$ circulan $^{2}$ a través de la correspondencia, y no faltan los enemigos financieros, concretamente la familia Schetz, señores de Grobbendonck, contra los que pleitea en España ${ }^{93}$. La nobleza es un cliente más de fiar que el clero, fiándonos de los beneficios obtenidos del préstamo a casas italianas y del Imperio (los Torello y los Neuhausen especialmente). Que de eso se escaparan acusaciones de usura es casi normal, considerando que los tipos de interés ofrecidos diferían mucho según lugar, tiempo y rango social ${ }^{94}$.

${ }^{86}$ MARTÍNEZ RUIZ, J. I., «The credit market and profits from Letters of Exchange. Ricorsa and exchange operations between Seville and the Besançon International Fairs (1589-1621)», The Journal of European Economic History, 33, 2 (2004), p. 339.

${ }^{87}$ Los Torello eran una importante familia parmesana. El clan de los Pallavicino estaba extendido por el norte de Italia, pero los que aparecen en la correspondencia (Giberto Pallavicino y su hijo Girolamo, que se educa en Flandes), son asimismo de Parma, AGRB, PLM, leg. 9, fols. 47-48.

88 AGRB, PLM, leg. 9, fol. 476. Carta de Struzzi, Bruselas, 22 de enero de 1609.

89 Struzzi pide consejo a Serra sobre los cambios vigentes en Piacenza porque «io non sono praticho nel cambio per detta piazza», ibid. Serra, como italiano, estaba muy vinculado a los negocios de la monarquía hispana; RODRÍGUEZ VILLA, A., Ambrosio Spínola..., pp. 151-152.

90 El fin de la tregua de doce años dificultó aún más el cobro. Hay un amplio dossier relativo a las deudas contraídas con Struzzi en AGS, CCGG, leg. 200-1, sin fol.

91 Struzzi tuvo negocios con los Fúcares a través de su agencia en Madrid, AGRB, PLM, leg. 9, fol. 129. Aunque exceptuados del decreto de suspensión de 1607, las condiciones de renegociación de la deuda fueron muy desventajosas para la casa; VOLTES, P., «Aportación a la historia de las operaciones de los Fugger en España durante el siglo XVII», Moneda y Crédito, 76 (1961), p. 26.

92 AGRB, PLM, leg., 9, fols. 471-472.

93 Ibid., fol. 95. Los Schetz, originarios de Maastricht, fueron banqueros de Carlos V. Erasmo, fundador de la firma, recibió en compensación la señoría de Grobbendonck. Participaron activamente a favor del rey en la guerra civil flamenca; VÁZQUEZ DE PRADA, V., Lettres marchandes..., tomo I, pp. 184185; KHEVENHÜLLER, H., Diario, Madrid, Sociedad Estatal para la Conmemoración de los Centenarios de Felipe II y Carlos V, 2001, pp. 340-341; DEL RíO, M. A., op. cit., pp. 116, 129, 148 y 153.

94 Si bien Struzzi concierta con Juan Enrique de Neuhausen un asequible 6 por ciento (AGRB, PLM, leg. 9, fol. 318, Praga, 13 de septiembre de 1603), gravará con un 30 por ciento a los Torello, quienes le acusan de «maligno y usurero» ante el duque de Parma (memorial de Francesco Torello, ibid., fol. 380, hacia 1608). Quizá todo obedeciera a la diversidad de mercados y de coyunturas. 
El aspecto financiero se complementa con el arrendamiento de algunas aduanas, operación factible durante la tregua de los doce años ${ }^{95}$. Struzzi efectúa asimismo negocios mercantiles sobre productos al por mayor y de lujo. En calidad de mayorista, contrata el abastecimiento de varias unidades del ejército ${ }^{96}$. Luego encontramos diversas partidas de textiles ${ }^{97}$. Con todo, se hace obvio que Struzzi trafica mayoritariamente con productos de alto valor añadido: ropa (vestidos especialmente), libros y bibliotecas ${ }^{98}$, cuadros, lámparas y objetos de iluminación, instrumentos musicales, tapicerías, y hasta el famoso ejército de juguete. Suelen aparecer asimismo las ventas de telas y botones de oro, donde Italia tenía la primacía incontestable y que se distribuían por todo el continente ${ }^{99}$; Londres aparece como mercado usual para la venta de dichos productos ${ }^{100}$. Aunque fuera de esta correspondencia, preciso es consignar que Struzzi fue un hombre muy interesado en las manufacturas, proveyendo de materias primas y tecnología allí donde le fueron solicitadas; además, presionó en ocasiones para la apertura de mercados a las industrias, sin lo cual cualquier iniciativa sería vana ${ }^{101}$. Ninguna de estas actividades empañaba su categoría de noble ${ }^{102}$.

\section{CONCLUSIONES}

Varias grandes conclusiones creemos desgajar del examen de las fuentes. La primera, que por sus orígenes y trayectoria vital, Alberto Struzzi era un hombre de ciudad: comercio, finanzas y manufacturas son actividades eminentemente urbanas. No encontramos en él las preocupaciones del hombre rural, aunque sabe muy bien que del campo depende en última instancia la buena o mala marcha de lo económico ${ }^{103}$.

95 Se trata del tonlieu de Zelanda, arrendado por un valor de 300 florines; AGRB, PLM, leg. 9, fol. 96. El tonlieu era un derecho de tránsito fluvial sobre los ríos del País Bajo.

96 AGRB, PLM, leg. 9, fols. 2-3, Asensio de Eguiguren a Struzzi, 26 de mayo de 1599. El contrato se hizo por un total de 909 florines y 8 placas, y Eguiguren, contador del ejército, pide a Struzzi la remisión de los detalles contables. Sobre estas banderas, O'DONNELL, H., La fuerza de desembarco de la Gran Armada contra Inglaterra (1588), Madrid, Ed. Naval, 1989, p. 161.

97 Melchior van Veltruyck, asociado de Struzzi, se dedica a la venta de «drappi de sete et altri», AGRB, PLM, leg. 9, fol. 21, Milán, 24 de noviembre de 1604.

98 Parece que sus asociados le informaban sobre bibliotecas en venta, ibid., fol. 187, carta de Antonio Boutappel, año 1614. Las clases pudientes adquirían a menudo libros. El emperador Rodolfo II o el rey Felipe II de España fueron grandes compradores de impresos y manuscritos, pero otros muchos príncipes se apuntaron a la moda, pues la guerra y las herencias eran buenas provisoras de material; ECHEVARRÍA BACIGALUPE, M. A., Vida y muerte..., pp. 320-325.

99 DE ROSA, L., «Introduzione», en II Mezzogiorno agli inizi del Seicento (L. De Rosa, Ed.), Bari, Laterza, 1994, p. XXXII.

100 AGRB, PLM, leg. 9, fols. 181 y 185.

101 La vertiente industrial de Struzzi, en ECHEVARRÍA BACIGALUPE, M. A., Alberto Struzzi..., pp. 135-163.

102 En principio, negociar al por mayor o con productos de lujo estaba permitido a la nobleza italiana; WELCH, E., De compras en el Renacimiento, Valencia, Universitat de València, 2009, p. 69.

103 BNM, ms. 10.441, fol. 222: «Las cossas son las que causan alteración en los precios y no las monedas, la qual alteración procede... de la cosecha». 
Comprobamos igualmente que las ciudades con las que se relaciona son urbes relevantes en el orden intelectual: Londres, Amberes, Lovaina, Nuremberg, Praga, Milán, Nápoles o el Madrid del Siglo de Oro, formaban parte de la gran red cultural europea, lo que nos explica muchas peculiaridades de su trayectoria ideológica, en especial el internacionalismo y la libertad de acción económica.

Otra nota a señalar es lo mucho que se involucra en temas económicos la nobleza media de Italia y los Países Bajos. A pesar del desprecio que sienten determinados grupos por el modo de vida de las clases inferiores nobiliarias ${ }^{104}$, ese colectivo jugó un papel relevante para la economía del capitalismo preindustrial, similar al que jugará la burguesía durante la industrialización ${ }^{105}$.

Acabaremos nuestro recorrido con una postrer consideración. El Struzzi huraño, enojado y contestatario que vemos en Madrid en los años veinte y treinta, no se corresponde en absoluto con este hombre dotado de una amplísima red de sociabilidad e intereses. ¿Qué ha podido cambiar? Primero, sus circunstancias personales, pues en la corte española no alcanza ni de lejos la relevancia que en Bruselas ${ }^{106}$. Luego, el triunfo de la vía belicista con la liquidación de la tregua en 1621 le pone en contra de los gobernantes. Su muerte en 1638, y la de Puteano en 1646, ponen fin a una generación que creyó que la paz perpetua emanaba necesariamente de la comunicación, que las actividades económicas eran la mejor vía para relacionarse, y que el poder del Príncipe no había de interferir en las estrategias de los particulares. 